\title{
Relationship between cognitive impairment and white-matter alteration in Parkinson's disease with dementia: tract-based spatial statistics and tract-specific analysis
}

\author{
Koji Kamagata • Yumiko Motoi • Hiroyuki Tomiyama • Osamu Abe • \\ Kenji Ito • Keigo Shimoji • Michimasa Suzuki • Masaaki Hori • \\ Atsushi Nakanishi • Tamotsu Sano • Ryohei Kuwatsuru • \\ Keisuke Sasai • Shigeki Aoki • Nobutaka Hattori
}

Received: 27 August 2012 /Revised: 4 December 2012 / Accepted: 20 December 2012 /Published online: 13 February 2013

(C) The Author(s) 2013. This article is published with open access at Springerlink.com

\begin{abstract}
Objectives We investigated the relationship between whitematter alteration and cognitive status in Parkinson's disease (PD) with and without dementia by using diffusion tensor imaging.

Methods Twenty PD patients, 20 PDD (Parkinson's disease with dementia) patients and 20 age-matched healthy controls underwent diffusion tensor imaging. The mean diffusivity and fractional anisotropy (FA) map of each patient group were compared with those of the control group by using tract-based spatial statistics. Tractography images of the genu of the corpus callosum fibre tracts were generated, and mean diffusivity and FA were measured.

Results FA values in many major tracts were significantly lower in PDD patients than in control subjects; in the prefrontal white matter and the genu of the corpus callosum they were significantly lower in PDD patients than in PD
\end{abstract}

K. Kamagata $(\bowtie) \cdot$ K. Ito $\cdot$ K. Shimoji $\cdot$ M. Suzuki $\cdot$ M. Hori $\cdot$

A. Nakanishi $\cdot$ R. Kuwatsuru $\cdot$ K. Sasai $\cdot$ S. Aoki

Department of Radiology, Juntendo University

School of Medicine, 2-1-1, Hongo, Bunkyo-ku,

Tokyo 113-8421, Japan

e-mail: kkamagat@juntendo.ac.jp

Y. Motoi $\cdot$ H. Tomiyama $\cdot$ T. Sano $\cdot$ N. Hattori

Department of Neurology, Juntendo University

School of Medicine, 2-1-1, Hongo, Bunkyo-ku,

Tokyo 113-8421, Japan

O. Abe

Department of Radiology, Nihon University School of Medicine,

30-1 Oyaguchi-Kamicho, Itabashi-ku,

Tokyo 173-8610, Japan patients. There was a significant correlation between the Mini-Mental State Examination (MMSE) scores and the FA values of the prefrontal white matter and the genu of the corpus callosum in patients with PD.

Conclusions Our study shows a relationship between cognitive impairment and alteration of the prefrontal white matter and genu of the corpus callosum. These changes may be useful in assessing the onset of dementia in PD patients.

Key Points

- Dementia is a common and important non-motor sign of Parkinson's disease (PD).

- The neuropathological basis of dementia in PD is not clear.

- DTI shows abnormalities in the prefrontal white matter in $P D$ with dementia.

- Prefrontal white matter alteration may be useful biomarker of dementia in PD.

Keywords Dementia - Diffusion tensor imaging ·

Parkinson's disease · Tract-based spatial statistics ·

Tract-specific analysis

$\begin{array}{ll}\text { Abbreviations } \\ \text { DTI } & \text { Diffusion tensor imaging } \\ \text { FA } & \text { Fractional anisotropy } \\ \text { HSD } & \text { Honestly significant difference } \\ \text { MD } & \text { Mean diffusivity } \\ \text { MMSE } & \text { Mini-Mental State Examination } \\ \text { PD } & \text { Parkinson's disease } \\ \text { PDD } & \text { Parkinson's disease with dementia } \\ \text { TBSS } & \text { Tract-based spatial statistics }\end{array}$




\section{Introduction}

Dementia, one of the most common and important nonmotor signs of Parkinson's disease (PD), greatly affects functioning and quality of life [1]. Cognitive impairment in PD occurs even at the early stage [2], and a recent longitudinal study that followed newly diagnosed PD patients for 20 years suggests that up to $80 \%$ will develop dementia [3]. However, despite the clinical importance of dementia, its neuropathological basis in PD remains controversial [4].

According to the hypothesis proposed by Braak et al. [5], deposition of Lewy bodies and Lewy neurites, which pathologically characterises PD, begins in the brain stem, spreads to the forebrain and limbic system, and finally reaches the neocortex. The pathological characteristics are divided into six subgroups according to the deposition of the Lewy bodies. These stages and the progression of cognitive impairment occur in parallel [6].

Many demographic and clinical characteristics have been evaluated as potential risk factors for the development of dementia in PD. Advanced age, relatively severe parkinsonism and mild cognitive impairment [7] have been identified as risk factors in several longitudinal studies [8]. Although demographic and clinical characteristics can be used to help estimate variations in the rate of cognitive decline and time to dementia in PD, objective biomarkers (such as magnetic resonance imaging [MRI] findings) are also needed.

In structural MRI studies, patients who have Parkinson's disease with dementia (PDD) have atrophy of the parietotemporal lobe, entorhinal cortex, hippocampus, prefrontal cortex and posterior cingulate, unlike PD patients without dementia or healthy controls [9-11].

There have also been reports of a relationship between cognitive status and white-matter alteration as determined by using diffusion tensor imaging (DTI). DTI can be used to measure the characteristics of local microstructural water diffusion in brain tissue. One representative diffusion tensor parameter is FA, which describes the degree of water molecule anisotropy or the directional preference of the water diffusion process. FA values in white matter are determined mainly by the cytoskeleton of the neurofilaments and microtubules and by the axonal membrane [12]. FA abnormalities in the brain are interpreted pathologically as being caused by loss of neurons or glia, by gliosis or demyelination [13], or by loss of the structural integrity of the white matter (i.e. white-matter damage) [14] Wiltshire et al. [15] reported a significant correlation between Mini-Mental State Examination (MMSE) score and mean diffusivity (MD) within the corpus callosum, Matsui et al. [16] reported that decreased fractional anisotropy (FA) values in the parietal white matter are associated with executive dysfunction, and Hattori et al. [14] reported that FA values in the parietal white matter significantly correlated with MMSE scores in patients with PD.

Wiltshire et al. [15] used tractography in their analysis, whereas Matsui et al. [16] used the region of interest (ROI) method. Both tractography and the ROI method have limitations in that their analytical domains are confined to fixed areas and it is difficult to prevent analyst bias. Hattori et al. used tract-based spatial statistics (TBSS) to perform a wholebrain analysis; for DTI they used 1.5-T MRI with a relatively small number of motion probing gradient (MPG) axes [12].

From these previous studies, we hypothesised that the changes in white matter in PDD patients are linked to cognitive impairment. We therefore performed a wholebrain analysis by using TBSS; for the DTI we used 3-T MRI, and we increased the number of MPG axes to 32 in an effort to overcome the limitations of previous studies.

After detecting specific white-matter alterations by using the TBSS method, we performed a more detailed investigation of the alteration by using tractography.

\section{Materials and methods}

\section{Subjects}

This study was approved by an institutional review board; informed consent was obtained from all participants before evaluation. For subjects who were deemed by the clinician not to have the capacity to give consent, a legally authorised surrogate provided consent.

There were 60 subjects: 20 PD patients, 20 PDD patients and 20 healthy controls. Note that some of these patients had also participated in our previous study [17]. All PD patients had been diagnosed by neurologists and met the United Kingdom Parkinson's Disease Society Brain Bank criteria [18]. PD was staged according to the Hoehn and Yahr scale, and the subjects underwent the Japanese version of the MMSE to assess cognitive dysfunction [19]. PDD was diagnosed in accordance with recently published guidelines [20], and patients with probable PDD were selected. All PD and PDD patients were taking levodopa (Table 1) at the time of the MRI and clinical examination.

Twenty-four months or more after MRI, all of the patients remained free of atypical parkinsonism and continued responding satisfactorily to anti-parkinsonian therapy. The 20 normal control subjects were recruited from the general population and were carefully matched by age with the patients. Individuals with any history of hypertension, diabetes mellitus, cardiovascular disease, stroke, brain tumour, epilepsy, Parkinson's disease, dementia, depression, drug abuse or head trauma were excluded as controls.

The demographic characteristics of the subjects are shown in Table 1. 
Table 1 Demographic characteristics of the subjects

\begin{tabular}{lllll}
\hline & Control $(n=20)$ & PD $(n=20)$ & PDD $(n=20)$ & $P$ value \\
\hline Sex, male : female & $10: 10$ & $8: 12$ & $10: 10$ & NS \\
Age in years, mean (SD) & $72.7(3.3)$ & $71.6(4.3)$ & $71.7(5.3)$ & NS \\
Disease duration in months, mean (SD) & NA & $04.0(53.4)$ & $146.6(91.0)$ & $<0.05$ (PD vs PDD) \\
Hoehn-Yahr stage (SD) & 28.8 & $2.4(1.0)$ & $2.9(0.7)$ & $<0.05$ (PD vs PDD) \\
MMSE (SD) & 0 & $25.7(3.2)$ & $18.7(5.8)$ & $<0.01$ (PD vs PDD) \\
Levodopa dosage mg/day, median (SD) & & $464.2(175.0)$ & $673.3(120.0)$ & $<0.05$ (PD vs PDD) \\
\hline
\end{tabular}

$P D$ Parkinson's disease, $P D D$ Parkinson's disease with dementia, MMSE Mini-Mental State Examination, NA not applicable, NS not significant $(P>0.05)$

${ }^{a}$ MMSE scores were significantly lower in PDD patients than in controls $(P<0.01)$ or PD patients $(P<0.01)$, but the scores did not differ significantly between controls and PD patients

MRI

MRI was performed at 3-T (Achieva; Philips Medical Systems, Best, The Netherlands) using an eight-channel phasedarray head coil for sensitivity encoding parallel imaging. Conventional MR images, such as T1-weighted spin echo images, T2-weighted turbo-spin echo images and fluidattenuated inversion recovery images, were obtained before acquisition of the diffusion tensor images. DTI was performed by using the spin-echo echo-planar technique (TR/TE $5,443 / 70 \mathrm{~ms}$, matrix size $128 \times 128$, field of view $224 \times$ $224 \mathrm{~mm}^{2}$, slice thickness $3 \mathrm{~mm}$ with no gap). Images were obtained with both 32-direction diffusion-encoding ( $b=$ $1,000 \mathrm{~s} / \mathrm{mm}^{2}$ for each direction) and no diffusion encoding $\left(b=0 \mathrm{~s} / \mathrm{mm}^{2}\right)$. A total of 50 axial section images were obtained, covering the whole cerebrum. MR data acquisition time was 7 min $17 \mathrm{~s}$.

\section{DTI image processing using TBSS}

Voxel-based analysis of the DTI data was performed using TBSS (tract-based spatial statistics) [21] implemented with the FMRIB Software Library 4.1.5 (FSL, Oxford Centre for Functional MRI of the Brain, UK; www.fmrib.ox.ac.uk/fsl), After eddy current correction and brain extraction had been performed, FA and MD images for all subjects were created by fitting a tensor model to the raw diffusion data. FA images of all subjects were aligned in standard Montreal Neurological Institute (MNI152) space by using FNIRT, a non-linear registration tool that uses a B-spline representation of the registration warp field .

Next, the mean FA image was created and thinned to create the mean FA skeleton, which represented the centres of all tracts common to the groups. The mean FA skeleton was thresholded to FA $>0.20$ to include the major whitematter pathways but exclude peripheral tracts and grey matter. Each subject's aligned FA map was then projected onto this skeleton by assigning to each point on the skeleton the maximum FA in a plane perpendicular to the local skeleton structure. The resulting skeletons were fed into voxel-wise statistics.

By applying the original non-linear registration of each subject's FA to the standard space, the MD was also projected onto the mean FA skeleton. The MD data were also used to calculate voxel-wise statistics.

Voxel-wise statistics of the skeletonised FA data were applied by using Randomise (part of FSL) to test for group differences between the patient and control groups. Randomise performed permutation-based testing with 5,000 permutations and inference by using TFCE (threshold-free cluster enhancement) [22] with a threshold of $P<0.05$, corrected for multiple comparisons by using the family-wise error correction.

Randomise was also used to examine the relationship between FA/MD and MMSE score, disease duration, Hoehn-Yahr stage, levodopa dosage in the PD and PDD patients group separately and combined, by using multiple linear regression analysis $(P<0.05$, corrected for gender and age at the time of MRI).

The anatomical locations of regions with significant group differences in FA and MD on the white-matter skeleton were identified by using a white-matter atlas [23].

DTI image processing using tract-specific analysis

DTI data were transferred to an offline workstation. Maps of FA and MD were computed by using dTV II and VOLUMEONE 1.72, developed by Masutani et al. [24] (University of Tokyo; diffusion tensor visualiser available at http:// www.volume-one.org/), Diffusion tensors were computed, and fibre tracts were created by using interpolation along the $\mathrm{z}$-axis to obtain data (voxel size $2.0 \times 2.0 \times 3.0 \mathrm{~mm}^{3}$ ). Colourcoded maps were created by using 33 sets of images ( 32 sets with $b=1,000 \mathrm{~s} / \mathrm{mm}^{2}, 1$ set with $b=0 \mathrm{~s} / \mathrm{mm}^{2}$ ). On the colour maps, red was assigned to left-right, green to anteroposterior and blue to the craniocaudal direction. Fibre tracts were based on the fibre assignment made by using the continuous tracking 
approach [25] to obtain a three-dimensional tract reconstruction. Identification of fibre tracts was initiated by placing a seed area in anatomical regions through which the particular fibres were expected to course [26]. Tract measurements were performed by two of the authors (K.S., K.K.), who were blinded to the disease status of the subjects.

Tractography of the genu of the corpus callosum was performed according to Hofer and Frahm's scheme [27], as the most anterior segment covers the first sixth of the corpus callosum and contains fibres projecting into the prefrontal region (Hofer and Frahm defined CC1). The seed ROI was placed manually, including the entirety of the genu of the corpus callosum (light blue area), on a reconstructed midsagittal image with a non-diffusion-weighted image $(b=$ $0 \mathrm{~s} / \mathrm{mm}^{2}$ ) (Fig. 1a). Tractographic images of the genu of the corpus callosum were generated with threshold values of linetracking termination FA $>0.18$ (Fig. 1b). The genu of the corpus callosum fibre tracts was defined as follows: anterior-posterior, including the first sixth of the corpus callosum; above and below, including the upper and lower border of the genu of the corpus callosum; and right-left, between the anterior horns of the lateral ventricles (Fig. 1c, d). Voxelisation along the genu of the corpus callosum was then performed. To reduce the partial volume effect of the peripheral portion of the tract and to eliminate small incidental artefactual lines, we used a shapeprocessing technique based on mathematical morphology [28]. In this shape-processing technique, we dilated the voxels once and eroded twice. FA and MD values in co-registered voxels were calculated (Fig. 1e).

\section{Statistical analysis}

Statistical analysis of demographic and clinical data was performed by using analysis of variance with Tukey's HSD (honestly significant difference) test for continuous variables and a $\chi^{2}$ test for categorical data. The criterion of statistical significance was $P<0.05$. Statistical analyses were performed with SPSS for Windows, Release 8.0.

We used the Kolmogorov-Smirnov test for analysis of normal distribution. Because only the MD of the PD group was not normally distributed, we used non-parametric (Kruskal-Wallis) analysis of variance and the Mann-Whitney $U$ test to test for differences between groups. A Bonferroni correction was applied for the number of comparisons $(n=3$ : [normal vs PD, normal vs PD, PD vs PDD], setting the level of significance at $P<0.05 / 3=0.016$ ).
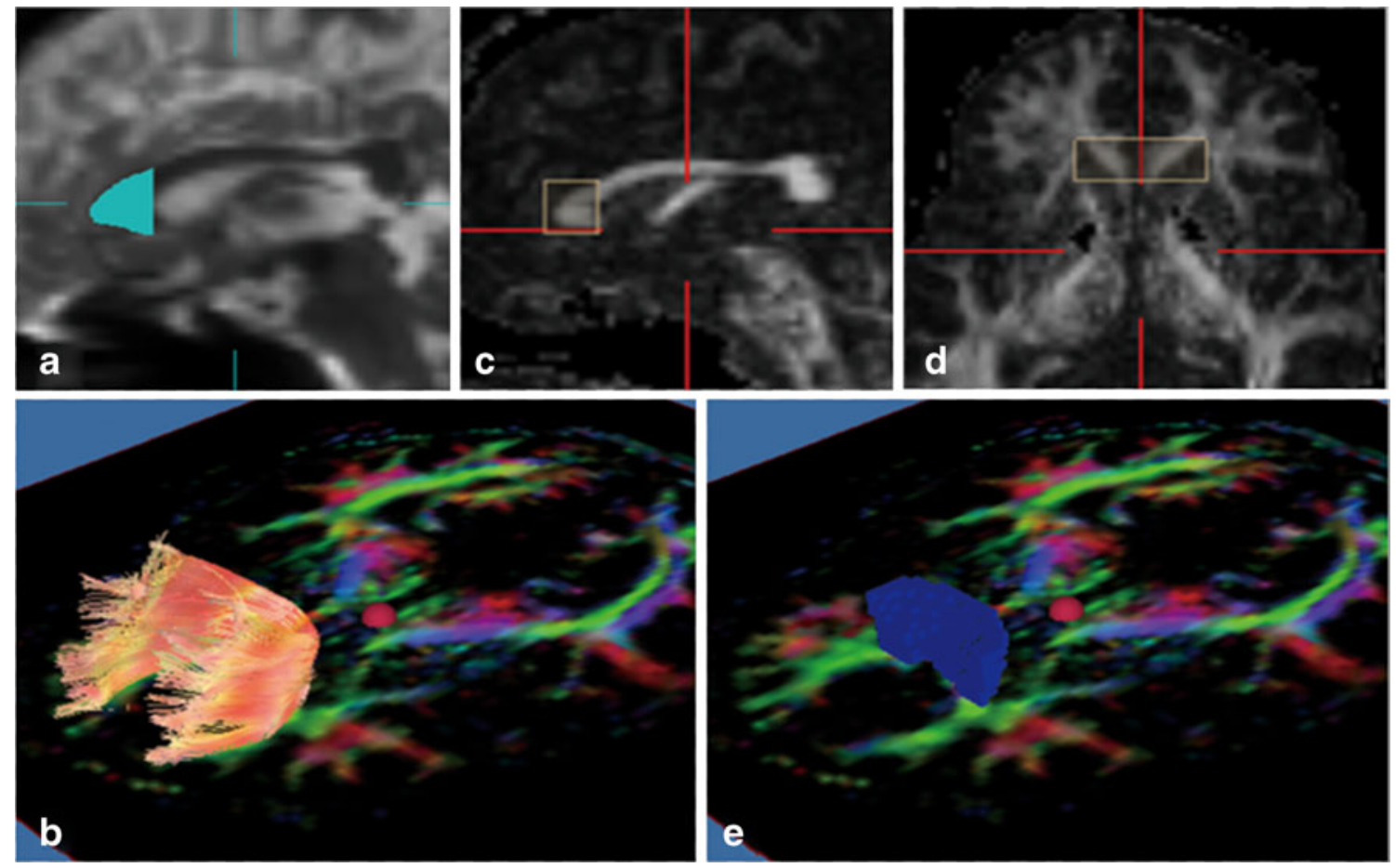

Fig. 1 Diffusion tensor tractography images of the genu of the corpus callosum and fibre tracts, and voxelisation. a The seed region of interest was placed manually including the entire genu of the corpus callosum (light blue area) on a reconstructed mid-sagittal image with a non-diffusion-weighted image $\left(b=0 \mathrm{~s} / \mathrm{mm}^{2}\right)$. b Tractographic image of the genu of the corpus callosum was generated with threshold values of line-tracking termination as fractional anistropy $(F A)>0.18$. c, d Fibre

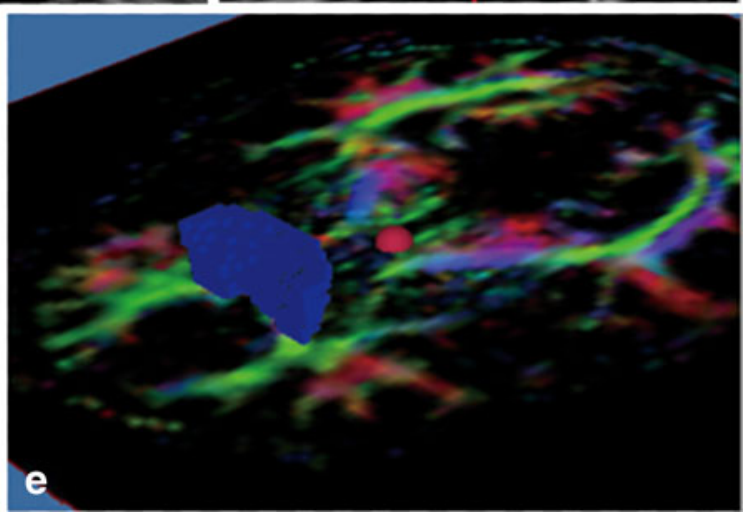

tracts of the genu of the corpus callosum were defined as follows: anterior-posterior, including the first sixth of the corpus callosum; above and below, including the upper and lower border of the genu of the corpus callosum; right-left, between the anterior horns of the lateral ventricles. e Voxelisation was performed along the genu of the corpus callosum (blue voxels). FA and mean diffusivity (MD) values in co-registered voxels were calculated 
Spearman's rank-order correlation test was used to investigate correlations between the imaging measurements and continuous clinical variables.

\section{Results}

Demographic and clinical features

The three groups did not differ by age ( $P>0.70$, ANOVA) or gender $\left(P>0.76, \chi^{2}\right)$ (Table 1). As expected, PDD patients scored significantly lower than PD patients and control subjects on the MMSE $(P<0.01, P<0.01$, ANOVA with Tukey's HSD test), but PD and control subjects did not differ significantly on the MMSE. There were significant differences in disease duration, Hoehn-Yahr stage, and levodopa dosage (mg/day) between PD and PDD patients (Table 1).
White-matter alteration assessed by using TBSS

FA and MD values were not significantly altered in the cerebral white matter of patients with PD compared with control subjects.

In patients with PDD, major white-matter tracts had significantly reduced FA values compared with the control group (Fig. 2a). The affected white-matter tracts included the superior and inferior longitudinal fasciculus, the inferior fronto-occipital fasciculus, the uncinate fasciculus, the cingulum, the anterior limb of the internal capsule and the substantia nigra. The reductions in FA were seen bilaterally in all of the affected white-matter tracts. Increased MD was found in major white-matter tracts in the PDD patients compared with the control group (Fig. 2b). MD was increased in almost the same white-matter tracts as those in which FA was decreased; in addition, MD was increased in the posterior limb of the internal capsule.

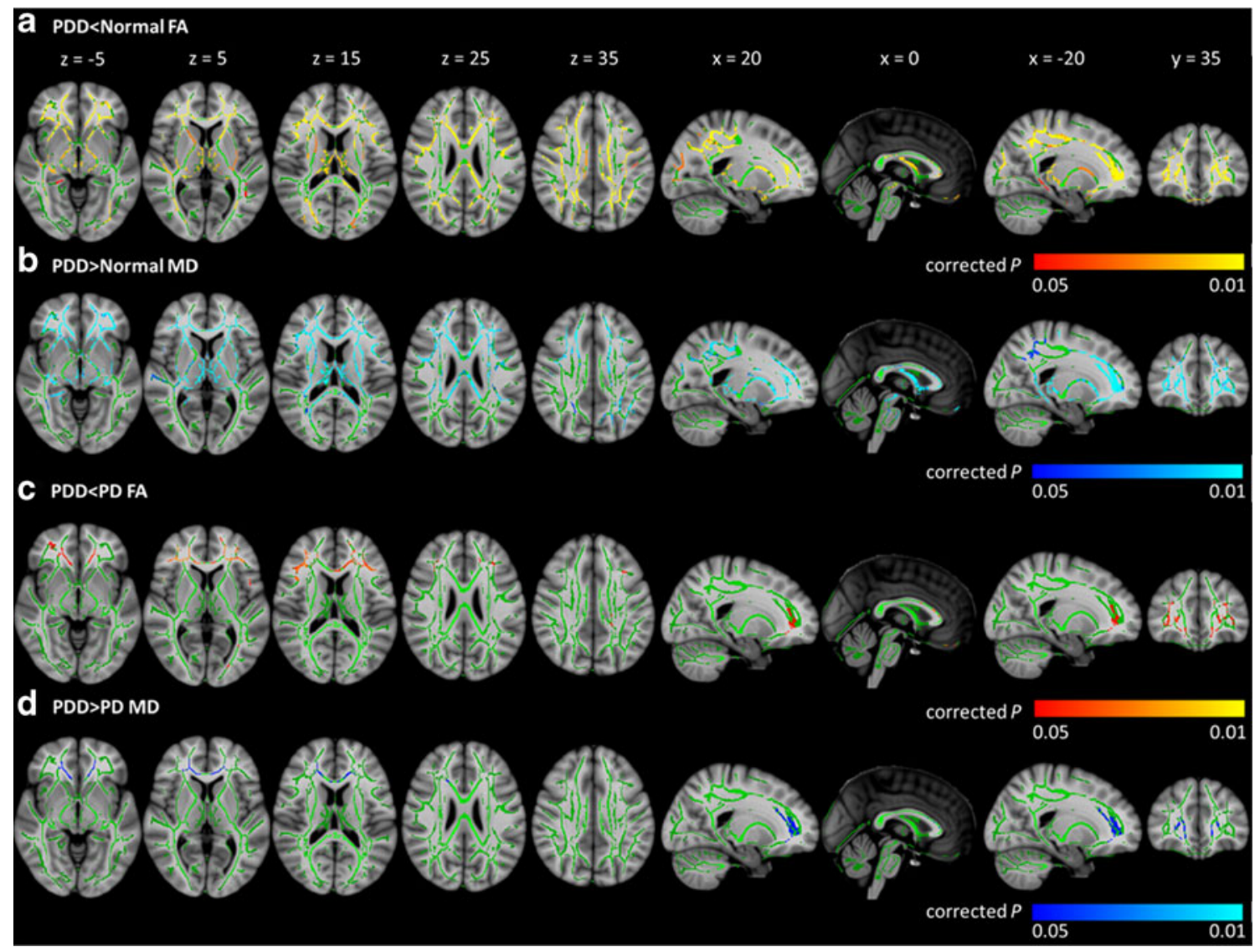

Fig. 2 Corrected $P$ maps. Tract-based spatial statistics (TBSS) analysis of the FA of normal control vs Parkinson's disease with dementia $(P D D)(\mathbf{a}), \mathrm{MD}$ of normal controls vs PDD (b), FA of Parkinson's disease $(P D)$ vs PDD (c), MD of PD vs PDD (d). All images are displayed in Montreal Neurological Institute space. a TBSS analysis demonstrated significantly decreased FA (red-yellow voxels) in several major white-matter tracts in the PDD group compared with the control group. b MD was increased in almost the same white-matter tracts as those in which FA was decreased in the PDD group compared with the control group (blue-light blue voxels). c In patients with PDD, FA values in the anterior part of the inferior fronto-occipital fasciculus (i.e. the white matter adjacent to the prefrontal area) and in part of the genu of the corpus callosum were significantly lower than those in PD patients (red-yellow voxels). d MD was increased in almost the same white-matter tracts as those in which FA was decreased in PDD patients relative to $\mathrm{PD}$ patients (blue-light blue voxels) 
In patients with PDD, FA values in the anterior part of the inferior fronto-occipital fasciculus (i.e. the white matter adjacent to the prefrontal area) and in part of the genu of the corpus callosum were significantly lower than in PD patients (Fig. 2c). MD was increased in almost the same white-matter tracts as those in which FA was decreased in PDD patients relative to PD patients (Fig. 2d).

In the PD and PDD groups combined, FA values correlated with MMSE scores. FA correlated positively with MMSE score in the anterior part of the inferior frontooccipital fasciculus, in part of the genu of the corpus callosum bilaterally, and in smaller areas in the right anterior and left posterior parts of the superior longitudinal fasciculus (precuneus) and in the left part of the corpus callosum (Fig. 3). FA and MD did not correlate with MMSE score in the separate PD and PDD groups. In the PD group, the PDD group, and the combined PD and PDD group, FA and MD did not correlate with disease duration, Hoehn-Yahr stage or levodopa dosage.

Because in PDD the FA values in the white matter adjacent to the prefrontal area and in part of the genu of the corpus callosum were significantly decreased and correlated positively with MMSE scores, we hypothesised that the genu of the corpus callosum, which contains fibres projecting into the prefrontal area, is involved in the pathological processes responsible for dementia in PD. We therefore compared diffusion abnormalities in the genu of the corpus callosum in PDD and PD patients with those in normal controls using diffusion tensor tractography.

White-matter alteration assessed by using tract-specific analysis

Reproducibility was determined on the basis of fibre counts and expressed as an intraclass correlation coefficient; the coefficient was 0.96 for the genu of the corpus callosum. Averaged values were therefore used for further statistical analyses.

There was a significant correlation between MMSE score and $\mathrm{FA}$ and $\mathrm{MD}$ values in the genu of the corpus callosum (FA: $r=0.472$; MD: $r=-0.453, P<0.005$ ) in the PD and PDD patient groups combined (Fig. 4). FA and MD did not correlate with MMSE score in the separate PD and PDD groups (FA in the PD group: $r=0.303, P=0.194$; MD in the PD group: $r=-0.165, P=0.488$; FA in the PDD group: $r=0.313, P=0.178$; MD in the PDD group: $r=-0.223, P=$ $0.344)$. However, we did observe a trend toward a positive correlation between FA in the genu of the corpus callosum and MMSE score. FA and MD values of the genu of the corpus callosum did not correlate significantly with disease duration or Hoehn-Yahr stage $(P>0.05)$ in patients with either PD or PDD.

FA and MD values in the genu of the corpus callosum were significantly lower in patients with PDD than in those with PD $(P=0.0050, P=0.0013)$ or in normal controls $(P=$ $0.0049, P=0.00014$ ) (Table 2 ). There were no significant differences in diffusion in the genu of the corpus callosum between PD patients and normal controls.

\section{Discussion}

Our study yielded three major findings. First, comparison of patients with PDD with a group of healthy individuals revealed diffusion abnormalities over a wide area of cerebral white matter. Second, a comparison of PDD patients with PD patients without dementia revealed diffusion abnormalities in the anterior superior longitudinal fasciculus (i.e. the cerebral white matter adjacent to the prefrontal area) and the genu of the corpus callosum. Third, multiple linear regression analysis
Fig. 3 In PD and PDD patients, TBSS analysis identified a significant positive correlation between FA and MMSE score. $(P<0.05$, corrected for multiple comparisons, gender and age at the time of MRI). Red-yellow voxels demonstrate a significant correlation between the MMSE score and FA

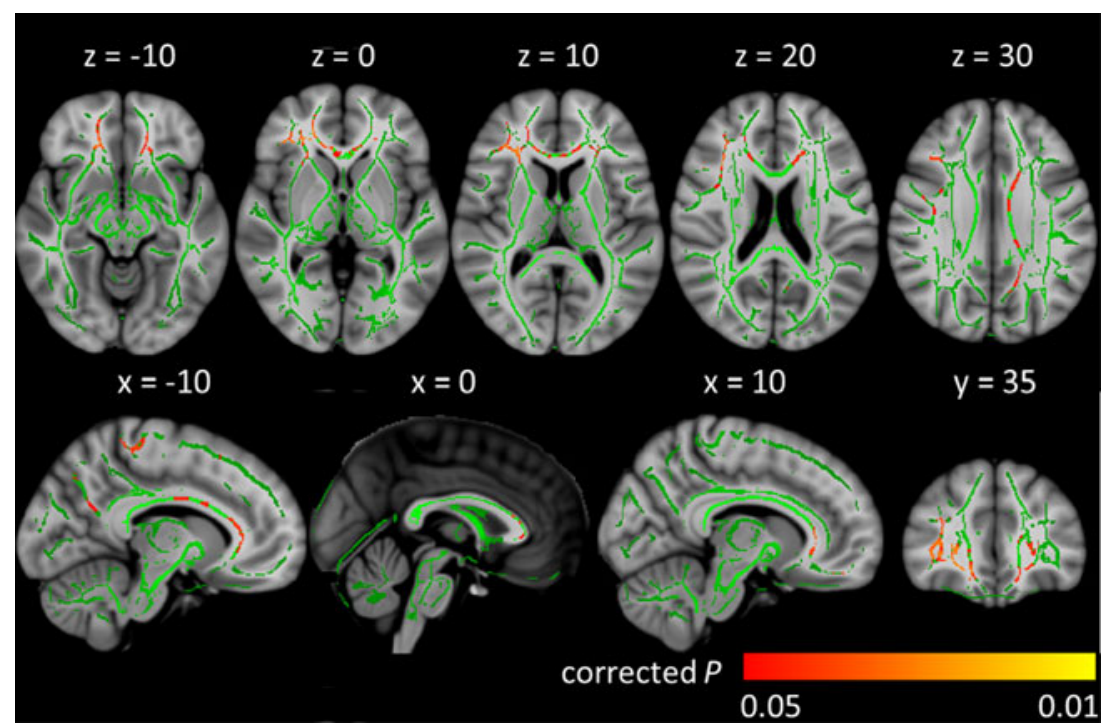



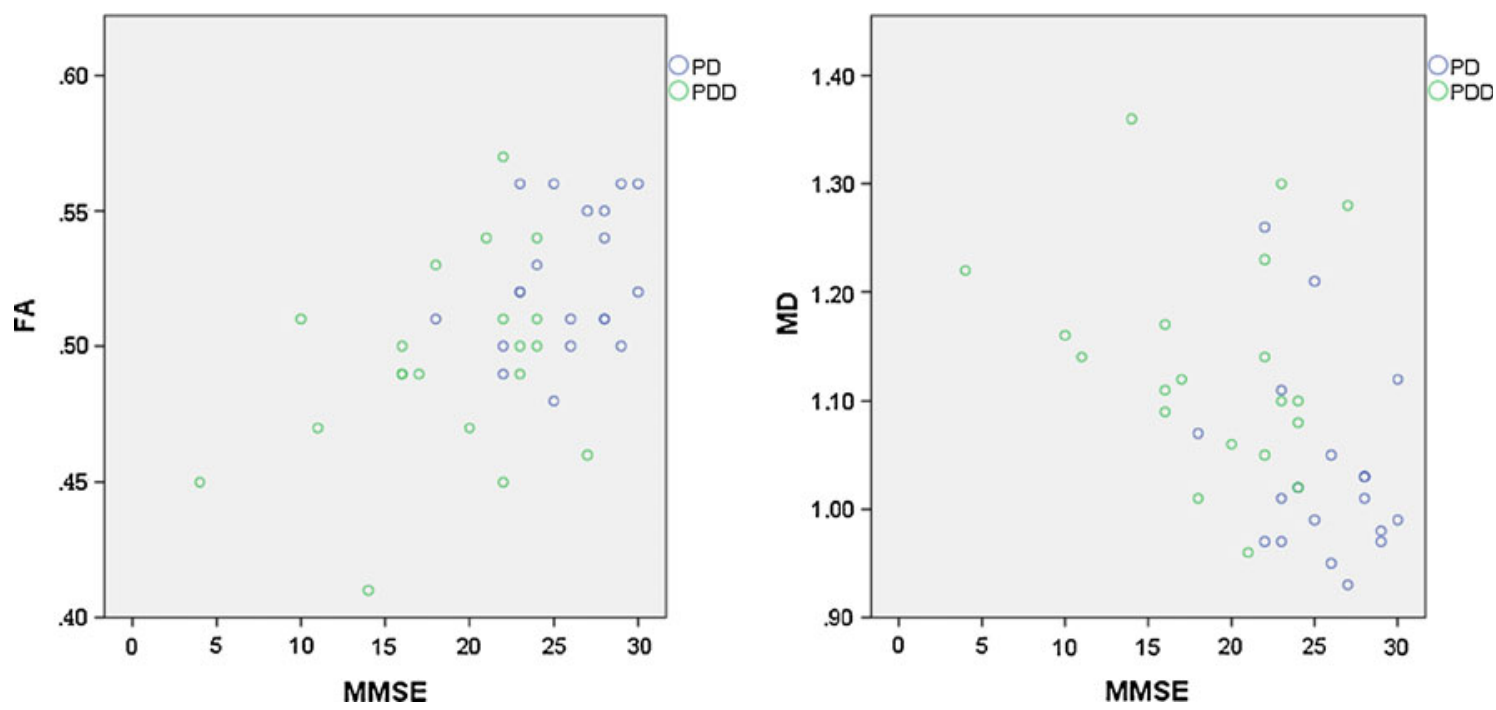

Fig. 4 FA and MD values in the tracts of the genu of the corpus callosum in the PD and PDD patient groups combined were significantly correlated with MMSE score (FA: $r=0.472, P=0.002$; MD: $\mathrm{r}=-0.453, P=0.003$ )

of TBSS revealed a significant correlation between the MMSE score and FA values of the cerebral white matter adjacent to the prefrontal area and the genu of the corpus callosum. Tract-specific analysis also detected a significant correlation between the FA values of the genu of the corpus callosum and the MMSE score, confirming the robustness of our results.

The widespread deterioration of cerebral white matter in the PDD group agrees with the results of the previous study by Hattori et al. [14]. Although the pathological features of white matter in PD are not well established, previous neuropathological studies suggest that white-matter damage is a multifaceted process. There are small Lewy abnormalities, such as Lewy neurites and Lewy axons, in the cerebral white matter in PD [5, 29]. Such accumulated Lewy abnormalities may have altered the axonal structure, resulting in FA values that were significantly lower in the major tracts in our PDD patients than in the controls.

Our comparison between the PDD and PD groups also revealed significant diffusion abnormalities in the genu of the corpus callosum and the white matter adjacent to the prefrontal area, uncovering a correlation between the MMSE score and the diffusion abnormality in these domains. These findings suggest that such changes could be used in assessing the onset of dementia in patients with PD.

However, FA and MD did not correlate with MMSE score in the separate PD and PDD groups when analysed by using TBSS and TSA. When we included both the PD and the PDD group combined, the FA values correlated positively with the MMSE scores in almost the same white matter (prefrontal white matter and genu of the corpus callosum) as that in which FA was decreased in PDD patients relative to PD patients. The finding that PDD patients had significantly lower MMSE scores than PD patients affected the result (FA values correlated positively with MMSE scores in the prefrontal white matter and the genu of the corpus callosum); however, the smaller patient number in this subgroup $(n=20,<40)$ may have influenced this finding.

There were significant differences in disease duration, Hoehn-Yahr stage, and levodopa dosage between the PD

Table 2 Comparison of mean diffusivity (MD) and fractional anisotropy (FA) in the genu of the corpus callosum in patients and control subjects

\begin{tabular}{|c|c|c|c|c|c|c|}
\hline & $\mathrm{CN}$ & $\mathrm{PD}$ & PDD & & & \\
\hline GCC & & & & $\mathrm{CN}>\mathrm{PD}$ & $\mathrm{CN}>\mathrm{PDD}$ & $\mathrm{PD}>\mathrm{PDD}$ \\
\hline \multirow[t]{2}{*}{ FA } & $0.52 \pm 0.03^{\mathrm{a}}$ & $0.52 \pm 0.03$ & $0.49 \pm 0.04$ & NS & $P<0.005^{\mathrm{c}}$ & $P<0.005^{\mathrm{c}}$ \\
\hline & & & & $\mathrm{CN}<\mathrm{PD}$ & $\mathrm{CN}<\mathrm{PDD}$ & $\mathrm{PD}<\mathrm{PDD}$ \\
\hline $\mathrm{MD}^{\mathrm{b}}$ & $1.01 \pm 0.07$ & $1.04 \pm 0.08$ & $1.13 \pm 0.10$ & NS & $P<0.00014^{\mathrm{c}}$ & $P<0.0013^{\mathrm{c}}$ \\
\hline
\end{tabular}

$G C C$ genu of the corpus callosum, $P D$ Parkinson's disease, $P D D$ Parkinson's disease with dementia, $C N$ control subjects

${ }^{\text {a }}$ Values are means \pm standard deviation

${ }^{\mathrm{b}}$ Diffusivity values are expressed as $10^{-3} \mathrm{~mm}^{2} / \mathrm{s}$

${ }^{\mathrm{c}}$ Statistical significance $(P<0.05)$ 
and PDD patients, and this may have affected the white matter alterations in the PDD group. However, FA and MD did not correlate with disease duration, Hoehn-Yahr stage, or levodopa dosage in the PD and PDD groups. This finding suggests that Hohen-Yahr stage, disease duration and levodopa dosage have little influence on white matter alteration compared with MMSE score.

The most consistent finding of structural MRI studies that have compared PDD patients with normal individuals or with PD patients has been atrophy of the entorhinal cortex, hippocampus, prefrontal cortex and posterior cingulate gyrus [9-11]. In recent studies in particular, atrophy of the white matter in the prefrontal area has been reported in addition to atrophy of the prefrontal cortex [30]. The pattern of atrophy of the entorhinal cortex, hippocampus and posterior cingulate gyrus is similar to the pattern reported in Alzheimer's disease, suggesting that Alzheimer-like disease abnormalities are part of the cause of the cognitive impairment in PD [31, 32]. The atrophy of the prefrontal cortex and white matter in PDD patients may also be related to the presence of Lewy abnormalities of the cortex and white matter. In fact, a neuropathological study has reported a link between the number of Lewy bodies in the cortex and the degree of dementia $[33,34]$. In the Braak stages, the deposition of Lewy bodies on the prefrontal cortex is classified as Stage 5 , which is a relatively early stage for the neocortex. Neurite abnormalities, parallel with Lewy bodies, are also found in PD [35]; these are called Lewy neuritis [36, 37]. Lewy abnormalities, such as Lewy neurites, are believed to spread to the adjacent white matter in addition to the prefrontal cortex. These pathological changes may be a cause of cognitive impairment in PD.

The corpus callosum is by far the largest fibre bundle in the human brain, interconnecting the two cerebral hemispheres with more than 300 million fibres and playing a primary role in both high-level cognitive integration and sensory integration [38]. Hofer and Frahm [27] defined five vertical partitions of the corpus callosum. Region I, the most anterior segment, covers the first sixth of the corpus callosum and contains fibres that project into the prefrontal region. The genu of the corpus callosum, which we defined here as equivalent to Region I, contains fibres that project into the prefrontal area [39]. In our study, the diffusion abnormalities of the genu of the corpus callosum therefore probably reflected prefrontal cortical Lewy abnormality and prefrontal white-matter damage.

Hattori et al. [14] compared a PDD group with a PD group and reported significant correlations between reduced FA and MMSE score in the superior and inferior longitudinal fasciculus, the lower frontal occipital fasciculus, the uncinate fasciculus, the cingulate fasciculus and the corpus callosum. They further reported significant correlations between the MMSE score and the decrease in FA values in the same domains. An examination of the figures included in their paper reveals that the alteration of the white matter was found largely in the parietal lobe. This finding differs considerably from our study results.

The difference between the results of our study and that of Hattori et al. may reflect the fact that our study used 3-T MRI and the diffusion tensor was imaged with a 32-axis MPG. Examinations of Monte Carlo simulations are said to require at least a 20-axis MPG to measure FA values and a 30-axis MPG to obtain more refined elements of the tensor [40]. Furthermore, in vivo examinations are said to require a 21-axis MPG for the calculation of FA values [41]. Thus the 12-axis MPG used in the study by Hattori et al. does not appear to meet these requirements; our study, which used a 32-axis MPG, may have produced more precise results. One limitation of our study, however, is that our patient group was smaller than that used by Hattori et al. Compared with 1.5-T MRI, 3-T MRI amplifies the distortion owing to the unevenness of the magnetic field. However, it produces high-resolution tensor images because of the increased SNR and a reduction in noise-related errors [42]. Compared with the PDD group in the report by Hattori et al. [14], our PDD group had a somewhat lower MMSE score; this may also have affected the results.

Hattori et al. reported significant correlations between reduced FA and MMSE score in the parietal lobe bilaterally, including in the precuneus; however, our results showed a significant correlation between the FA value of only the left side of the precuneus and the MMSE score. One possible reason why the right precuneus did not produce the same result could be that the FA changes in the precuneus were small and at the threshold value, i.e. the limit of detection (TFCE-corrected $P<0.05$ ). Figure 3 shows that major sections in the left precuneus are almost entirely red, indicating the significant correlation between MMSE and FA. Another possible reason why this change was found only on one side is that our results may reflect the laterality of cognitiverelated white alterations. Hence, further studies are needed to clarify whether cognitive impairment is associated with laterality of white matter alterations.

We previously reported reduced FA in the anterior cingulate fibre tracts in PD patients and a significant correlation between FA in the anterior cingulate fibre tracts and MMSE score by using tract-specific analysis in PDD patients [17]. In the present study, we detected reduced FA bilaterally in the cingulate fibre tracts of PDD patients compared with in normal controls, but we did not detect any reduced FA in PD patients or a significant correlation between FA in the anterior cingulate fibre tracts and MMSE score when we used TBSS in PDD patients.

Compared with TSA, TBSS can influence the outcome by multiple comparisons, whereas TSA has the advantage of detecting abnormalities in specific white matter tracts. 
Therefore, our use of TBSS may have been the reason why we did not detect reduced FA in the anterior cingulate fibre tracts in PD patients.

The limitations of our study need to be addressed. Because the diagnoses of PD and PDD were not histopathologically confirmed, the possibility of misdiagnosis remains. However, the validity of the diagnoses is supported by the finding that, 24 months or more after undergoing imaging, all of the patients continued to respond satisfactorily to anti-parkinsonian therapy and remained free of atypical parkinsonism.

In conclusion, we consider that the finding of diffusion abnormalities in the cerebral white matter of PDD patients reflects structural changes resulting from Lewy abnormalities in the cerebral white matter. Our study also found a relationship between cognitive impairment and alteration of white matter adjacent to the prefrontal area and in the genu of the corpus callosum. These changes may be useful in assessing the onset of dementia in PD patients.

Acknowledgments We thank Nozomi Hamasaki and Syuji Sato, MRI technologists, for their skilful performance in data acquisition; Toshino Suzuki, Tomomi Okamura and Yasmin Hasina for their research assistance; and Yuriko Suzuki and Masaru Takashima, Philips Healthcare for their technical assistance. We also thank Narisumi Cho for administrative assistance. This work was supported by a Grant-inAid for Scientific Research on Innovative Areas (Comprehensive Brain Science Network) from the Ministry of Education, Science, Sports, and Culture of Japan, and MEXT/JSPS KAKENHI Grant Number 24591787.

Note that some of these patients also participated in our previous study. However, overlap of the case is below a half and the main analysis methods in the present study differ from our previous report. Only the cingulate fibre tract was evaluated by tract-specific analysis in our previous study, but the main analysis techniques in the present study is a whole-brain analysis by using TBSS. Tract-specific analysis was used to confirm the robustness of our results in present study.

Open Access This article is distributed under the terms of the Creative Commons Attribution Noncommercial License which permits any noncommercial use, distribution, and reproduction in any medium, provided the original author(s) and the source are credited.

\section{References}

1. Winter Y, von Campenhausen S, Arend M et al (2011) Health-related quality of life and its determinants in Parkinson's disease: results of an Italian cohort study. Parkinsonism Relat Disord 17:265-269

2. Muslimovic D, Post B, Speelman JD, Schmand B (2005) Cognitive profile of patients with newly diagnosed Parkinson disease. Neurology 65:1239-1245

3. Hely MA, Reid WG, Adena MA, Halliday GM, Morris JG (2008) The Sydney multicenter study of Parkinson's disease: the inevitability of dementia at 20 years. Mov Disord 23:837-844

4. Svenningsson P, Westman E, Ballard C, Aarsland D (2012) Cognitive impairment in patients with Parkinson's disease: diagnosis, biomarkers, and treatment. Lancet Neurol 11:697-707

5. Braak H, Sandmann-Keil D, Gai W, Braak E (1999) Extensive axonal Lewy neurites in Parkinson's disease: a novel pathological feature revealed by alpha-synuclein immunocytochemistry. Neurosci Lett 265:67-69
6. Braak H, Rub U, Jansen Steur EN, Del Tredici K, de Vos RA (2005) Cognitive status correlates with neuropathologic stage in Parkinson disease. Neurology 64:1404-1410

7. Caviness JN, Lue L, Adler CH, Walker DG (2011) Parkinson's disease dementia and potential therapeutic strategies. CNS Neurosci Ther 17:32-44

8. Aarsland D, Kurz MW (2010) The epidemiology of dementia associated with Parkinson's disease. Brain Pathol 20:633-639

9. Burton EJ, McKeith IG, Burn DJ, Williams ED, O’Brien JT (2004) Cerebral atrophy in Parkinson's disease with and without dementia: a comparison with Alzheimer's disease, dementia with Lewy bodies and controls. Brain 127:791-800

10. Song SK, Lee JE, Park HJ, Sohn YH, Lee JD, Lee PH (2011) The pattern of cortical atrophy in patients with Parkinson's disease according to cognitive status. Mov Disord 26:289-296

11. Weintraub D, Doshi J, Koka D et al (2011) Neurodegeneration across stages of cognitive decline in Parkinson disease. Arch Neurol 68:1562-1568

12. Beaulieu C (2002) The basis of anisotropic water diffusion in the nervous system - a technical review. NMR Biomed 15: 435-455

13. Abe O, Aoki S, Hayashi $\mathrm{N}$ et al (2002) Normal aging in the central nervous system: quantitative MR diffusion-tensor analysis. Neurobiol Aging 23:433-441

14. Hattori T, Orimo S, Aoki S et al (2012) Cognitive status correlates with white matter alteration in Parkinson's disease. Hum Brain Mapp 33:727-739

15. Wiltshire K, Concha L, Gee M, Bouchard T, Beaulieu C, Camicioli $\mathrm{R}$ (2010) Corpus callosum and cingulum tractography in Parkinson's disease. Can J Neurol Sci 37:595-600

16. Matsui $H$, Nishinaka $K$, Oda $M$, Niikawa $H$, Kubori T, Udaka $F$ (2007) Dementia in Parkinson's disease: diffusion tensor imaging. Acta Neurol Scand 116:177-181

17. Kamagata K, Motoi Y, Abe O et al (2012) White matter alteration of the cingulum in Parkinson disease with and without dementia: evaluation by diffusion tensor tract-specific analysis. AJNR Am J Neuroradiol 33:890-895

18. Hughes AJ, Daniel SE, Kilford L, Lees AJ (1992) Accuracy of clinical diagnosis of idiopathic Parkinson's disease: a clinicopathological study of 100 cases. J Neurol Neurosurg Psychiatry 55:181-184

19. Folstein MF, Folstein SE, McHugh PR (1975) "Mini-mental state". A practical method for grading the cognitive state of patients for the clinician. J Psychiatr Res 12:189-198

20. Goetz CG, Emre M, Dubois B (2008) Parkinson's disease dementia: definitions, guidelines, and research perspectives in diagnosis. Ann Neurol 64:S81-S92

21. Smith SM, Jenkinson M, Johansen-Berg H et al (2006) Tract-based spatial statistics: voxelwise analysis of multi-subject diffusion data. Neuroimage 31:1487-1505

22. Nichols TE, Holmes AP (2002) Nonparametric permutation tests for functional neuroimaging: a primer with examples. Hum Brain Mapp 15:1-25

23. Mori S, Wakana S, Van Zijl PCM (2004) MRI atlas of human white matter. Elsevier, Amsterdam

24. Masutani Y, Aoki S, Abe O, Hayashi N, Otomo K (2003) MR diffusion tensor imaging: recent advance and new techniques for diffusion tensor visualization. Eur J Radiol 46:53-66

25. Mori S, Crain BJ, Chacko VP, van Zijl PC (1999) Threedimensional tracking of axonal projections in the brain by magnetic resonance imaging. Ann Neurol 45:265-269

26. Wakana S, Jiang H, Nagae-Poetscher LM, van Zijl PC, Mori S (2004) Fiber tract-based atlas of human white matter anatomy. Radiology 230:77-87

27. Hofer S, Frahm J (2006) Topography of the human corpus callosum revisited-comprehensive fiber tractography using 
diffusion tensor magnetic resonance imaging. Neuroimage 32: 989-994

28. Yasmin H, Nakata Y, Aoki S et al (2008) Diffusion abnormalities of the uncinate fasciculus in Alzheimer's disease: diffusion tensor tract-specific analysis using a new method to measure the core of the tract. Neuroradiology 50:293-299

29. Saito Y, Kawashima A, Ruberu NN et al (2003) Accumulation of phosphorylated alpha-synuclein in aging human brain. J Neuropathol Exp Neurol 62:644-654

30. Takahashi R, Ishii K, Miyamoto N et al (2010) Measurement of gray and white matter atrophy in dementia with Lewy bodies using diffeomorphic anatomic registration through exponentiated lie algebra: a comparison with conventional voxel-based morphometry. AJNR Am J Neuroradiol 31:1873-1878

31. Weintraub D, Dietz N, Duda JE et al (2012) Alzheimer's disease pattern of brain atrophy predicts cognitive decline in Parkinson's disease. Brain 135:170-180

32. Sabbagh MN, Adler CH, Lahti TJ et al (2009) Parkinson disease with dementia: comparing patients with and without Alzheimer pathology. Alzheimer Dis Assoc Disord 23:295-297

33. Hurtig HI, Trojanowski JQ, Galvin J et al (2000) Alpha-synuclein cortical Lewy bodies correlate with dementia in Parkinson's disease. Neurology 54:1916-1921

34. Aarsland D, Perry R, Brown A, Larsen JP, Ballard C (2005) Neuropathology of dementia in Parkinson's disease: a prospective, community-based study. Ann Neurol 58:773-776
35. Gai WP, Blessing WW, Blumbergs PC (1995) Ubiquitin-positive degenerating neurites in the brainstem in Parkinson's disease. Brain 118:1447-1459

36. Gai WP, Blumbergs PC, Blessing WW (1996) Microtubule-associated protein 5 is a component of Lewy bodies and Lewy neurites in the brainstem and forebrain regions affected in Parkinson's disease. Acta Neuropathol 91:78-81

37. Kanazawa T, Adachi E, Orimo S, Nakamura A, Mizusawa H, Uchihara T (2012) Pale neurites, premature alpha-synuclein aggregates with centripetal extension from axon collaterals. Brain Pathol 22:67-78

38. Gazzaniga MS (2000) Cerebral specialization and interhemispheric communication: does the corpus callosum enable the human condition? Brain 123:1293-1326

39. Witelson SF (1989) Hand and sex differences in the isthmus and genu of the human corpus callosum. A postmortem morphological study. Brain 112:799-835

40. Jones DK (2004) The effect of gradient sampling schemes on measures derived from diffusion tensor MRI: a Monte Carlo study. Magn Reson Med 51:807-815

41. Ni H, Kavcic V, Zhu T, Ekholm S, Zhong J (2006) Effects of number of diffusion gradient directions on derived diffusion tensor imaging indices in human brain. AJNR Am J Neuroradiol 27:1776-1781

42. Hunsche S, Moseley ME, Stoeter P, Hedehus M (2001) Diffusiontensor MR imaging at 1.5 and $3.0 \mathrm{~T}$ : initial observations. Radiology 221:550-556 